\title{
Role of the p38 MAPK signaling pathway in mediating interleukin-28A-induced migration of UMUC-3 cells
}

\author{
SE-JUNG LEE ${ }^{1-3}$, WUN-JAE KIM ${ }^{3}$ and SUNG-KWON MOON ${ }^{1,3}$ \\ ${ }^{1}$ Department of Biotechnology, Chungju National University, Chungju, Chungbuk 380-702; ${ }^{2}$ Department of Food Science and \\ Technology, Chung-Ang University, Ansung, Gyunggi-do 456-756; ${ }^{3}$ Personalized Tumor Engineering Research Center, \\ Department of Urology, Chungbuk National University, Cheongju, Chungbuk 361-763, Republic of Korea
}

Received March 24, 2012; Accepted June 13, 2012

DOI: $10.3892 /$ ijmm.2012.1064

\begin{abstract}
Although interleukin-28A (IL-28A) is believed to have an antiviral effect, its role in tumor migration requires further examination. The present study was intended to verify the effect of IL-28A on the migration of UMUC-3 bladder cancer cells. IL-28A and its receptor IL-28AR1 mRNA were detected in UMUC-3 cells. Although exogenous IL-28A showed no effect on cell proliferation, a wound-healing migration assay showed that the migration of UMUC-3 cells was induced by IL-28A. Furthermore, treatment of the cells with IL-28A significantly promoted MMP-9 expression via binding activities of NF- $\kappa$ B and AP-1. IL-28A also induced the activation of p38 MAPK and Jak2-Stat2 signaling. Using the p38 MAPK inhibitor SB203580 and the dominant-negative plasmid DN-p38, we found evidence that the inhibition of p38 MAPK signaling suppressed the effects of IL-28A including wound-healing migration and MMP-9 expression by activation of NF- $\kappa$ B and AP-1 binding in UMUC-3 cells. However, Jak-2 inhibition by AG490 did not affect IL-28Ainduced migration of UMUC-3 cells. Collectively, we suggest for the first time that the p38 MAPK pathway mediates IL-28A-induced cell migration through MMP-9 expression by activating $\mathrm{NF}-\kappa \mathrm{B}$ and $\mathrm{AP}-1$ binding motifs.
\end{abstract}

\section{Introduction}

Epidemiological studies have demonstrated that transitional cell carcinoma (TCC) of the bladder is a common urologic malignancy in men (1-3). Most of the deaths of patients with muscle invasive bladder cancer are due to the progression of their disease caused by metastasis $(2,4,5)$. Therefore, understanding these metastatic mechanisms is essential for the design of more effective therapeutic targets. Although many

Correspondence to: Dr Sung-Kwon Moon, Department of Biotechnology, Chungju National University, 123 Geomdan-ri Iryumyeon, Chungju, Chungbuk 380-702, Republic of Korea

E-mail: sumoon66@dreamwiz.com

Key words: interleukin-28A, bladder cancer cells, migration, p38 MAPK, matrix metalloproteinases-9 reports have studied cascading events leading to the metastasis of muscle invasive bladder tumors $(2,6)$, the exact molecular mechanisms responsible for bladder cancer metastasis remain poorly understood.

The metastatic process is associated with the migration and invasion of tumor cells, which requires the alteration of the extracellular matrix (ECM) and the basement membrane by action of proteinases such as the matrix metalloproteinases (MMPs) (7-9). MMP-2 (gelatinase A) and MMP-9 (gelatinase $\mathrm{B}$ ) are type IV collagenases that degrade the ECM and the basement membrane, resulting in the migration of tumor cells (7-9). Previous studies suggest that MMP-9 expression is closely correlated with the progression of muscle invasive bladder tumors $(10,11)$. The expression of MMP-9 was found to be induced by various growth factors, growth factor receptors, and cytokines via the binding of several transcription factors including AP-1, Sp-1 and NF- $\mathrm{B}$ (12-15). In addition, intracellular signaling, such as the MAPK and Jak-Stat signaling pathways, is known to be involved in the regulation of MMP-9 expression $(12,16)$.

Interleukin-28A (IL-28A) was originally identified as a human IFN- $\lambda 2$ protein $(17,18)$, and is a member of the IL-10 gene family $(17,18)$. Important signaling pathways, such as MAPK and Jak-Stat, are associated with IL-28A stimulation $(17,19)$. Moreover, IL-28AR1 is reportedly expressed in cancers of the bladder, blood, breast, brain, head, neck and lung (20). IL-28A interacts with the IL-28AR1 receptor leading to many biological activities including antiviral activity and inhibition of tumor growth (17-22). However, the molecular mechanisms of IL-28A in modulating the migration of human cancer cells remain elusive.

Thus, we attempted to elucidate the exact roles and molecular mechanisms of IL-28A in the migration of bladder cancer cells. In the present study, we demonstrated that IL-28A induces cell migration through p38 MAPK-dependent MMP-9 expression by activating transcription factors NF- $\kappa \mathrm{B}$ and $\mathrm{AP}-1$ in UMUC-3 bladder cancer cells.

\section{Materials and methods}

Materials. Polyclonal antibodies to ERK, phospho-ERK, p38 MAPK, phospho-p38 MAPK, JNK, and phospho-JNK were obtained from Cell Signaling Technology (Danvers, MA). 
Polyclonal antibodies to Jak1, Jak2, Jak3, Stat1, Stat2, Stat3, Stat5, phospho-Jak1, phospho-Jak2, phospho-Jak3, phosphoStat1, phospho-Stat2, phospho-Stat3, and phospho-Stat5 were purchased from Santa Cruz Biotechnology, Inc. (Santa Cruz, CA). SB203580, piceatannol, and AG490 were obtained from Calbiochem (San Diego, CA). The polyclonal MMP-9 antibody was obtained from Chemicon. Dominant negative p38 MAPK (DN-p38) was provided by Dr Roger Davis (University of Massachusetts Medical School, Worcester, MA).

Cell cultures. A human bladder carcinoma cell line (UMUC-3) was obtained from the American Type Culture Collection. The cells were maintained in Dulbecco's modified Eagle's medium (DMEM) (4.5 g glucose/l) supplemented with $10 \%$ fetal bovine serum (FBS), L-glutamine, and antibiotics (Biological Industries, Beit Haemek, Israel) at $37^{\circ} \mathrm{C}$ in a $5 \% \mathrm{CO}_{2}$ humidified incubator.

RNA extraction. Total-RNA was isolated from tissue using TRIzol reagent (Life Technologies, Grand Island, NY), according to the manufacturer's protocol. The quality and integrity of the RNA were confirmed by agarose gel electrophoresis and ethidium bromide staining, followed by visual examination under ultraviolet light.

Real-time polymerase chain reaction (PCR). Real-time PCR assays using a Rotor-Gene 3000 PCR system (Corbett Research, Mortlake, Australia) were performed in the original and independent cohorts. GAPDH was analyzed in parallel as an internal control. Real-time PCR reactions containing primers and SYBR Premix Ex Taq (Takara Bio Inc., Otsu, Japan) were carried out in micro-reaction tubes (Corbett Research). Spectral data were captured and analyzed using Rotor-Gene Real-Time Analysis Software 6.0 Build 14 (Corbett Research). For amplification, IL-28A, sense, 5'-ACC GCT GAC ACT GAC CCA G-3' and antisense, 5'-CAG CCA GGG GAC TCC TTT-3' primers or IL-28AR1, sense, 5'-CAG AAT GTG ACG CTG CTC TC-3' and antisense, 5'-ATC CAG GTA TTC GGA CTC CA-3' primers were used. GAPDH was analyzed in parallel as an endogenous RNA reference gene, and data were normalized to the expression of GAPDH.

Immunoblot analysis. Growth-arrested cells were treated with IL-28A in the absence of $10 \%$ FBS for various durations at $37^{\circ} \mathrm{C}$. The cells were then washed twice with cold phosphatebuffered saline (PBS) and freeze-thawed in $250 \mu 1$ lysis buffer (containing, in mmol/l, HEPES [pH 7.5] 50, NaCl 150, EDTA 1, EGTA 2.5, DTT 1, $\beta$-glycerophosphate $10, \mathrm{NaF} 1, \mathrm{Na}_{3} \mathrm{VO}_{4} 0.1$, and phenylmethylsulfonyl fluoride 0.1 and $10 \%$ glycerol, $0.1 \%$ Tween-20, $10 \mathrm{~g} / \mathrm{ml}$ of leupeptin, and $2 \mu \mathrm{g} / \mathrm{ml}$ of aprotinin), and then scraped into $1.5-\mathrm{ml}$ tubes. The lysates were placed on ice for $15 \mathrm{~min}$ and then centrifuged at $12,000 \mathrm{rpm}$ for $20 \mathrm{~min}$ at $4^{\circ} \mathrm{C}$. The protein concentration of the supernatant was determined using a Bradford reagent method (Bio-Rad). Equal amounts of cellular proteins were resolved by electrophoresis on a $0.1 \%$ SDS-10\% polyacrylamide gel (SDS-PAGE) under denaturing conditions. The proteins were transferred electrophoretically to nitrocellulose membranes (Hybond; Amersham Corp., Arlington Heights, IL). After blocking in $10 \mathrm{mmol} / \mathrm{l}$ Tris- $\mathrm{HCl}(\mathrm{pH} 8.0), 150 \mathrm{mmol} / \mathrm{l} \mathrm{NaCl}$, and 5\% (wt/vol) nonfat dry milk, the membranes were treated with primary antibodies for $90 \mathrm{~min}$, followed by incubation with peroxidase-conjugated secondary antibodies for $45 \mathrm{~min}$. The immunocomplexes were detected using a chemiluminescence reagent kit (Amersham Corp). For the immunoblotting studies, the experiments were repeated at least 3 times.

Wound-healing migration assay. Cells were plated on 6-well dishes and grown to $90 \%$ confluence in $2 \mathrm{ml}$ of growth medium. The cells were damaged using a 2-mm-wide tip and were then treated with IL-5. They were allowed to migrate, and images were captured using an inverted microscope ( $\mathrm{x} 40$ magnification).

MTT assay. The incorporation of 3-(4,5-dimethylthiazol-2-yl)2,5-diphenyltetrazolium bromide (MTT; Sigma-Aldrich) was used to assess cell proliferation. Briefly, 5,000 cells/well were seeded on a 96-well plate. After serum starvation for $24 \mathrm{~h}$, the cells were treated with the indicated concentrations of IL-28A and then further incubated for 4 days. MTT $(5 \mathrm{mg} / \mathrm{ml})$ was added, and the medium was removed $4 \mathrm{~h}$ later. The colored formazan product was resolved in $0.04 \mathrm{~N} \mathrm{HCl} /$ isopropanol and quantified by absorbance measurements at $570 \mathrm{~nm}$.

Zymography. Conditioned medium was electrophoresed in a polyacrylamide gel containing $1 \mathrm{mg} / \mathrm{ml}$ gelatin. The gel was then washed at room temperature for $2 \mathrm{~h}$ with $2.5 \%$ Triton X-100 and then soaked overnight at $37^{\circ} \mathrm{C}$ in a buffer containing $10 \mathrm{mM} \mathrm{CaCl}_{2}, 150 \mathrm{mM} \mathrm{NaCl}$ and $50 \mathrm{mM}$ Tris- $\mathrm{HCl}$, $\mathrm{pH}$ 7.5. The gel was stained with $0.2 \%$ Coomassie blue and photographed on a light box. Proteolysis was detected as a white zone on a dark blue field (15).

Transfection. Cells were transfected with DN-p38 using Lipofectamine 2000 transfection reagent according to the manufacturer's protocol (Invitrogen, Carlsbad, CA). After the indicated incubation with IL-28A, the cells were studied via immunoblotting, zymography, electrophoretic mobility shift assay (EMSA), and wound-healing migration assay.

Nuclear extracts and EMSA. Cultured cells were collected by centrifugation, washed, and suspended in a buffer containing $10 \mathrm{mM}$ 4-(2-hydroxyethyl)-1-piperazine ethanesulfonic acid (HEPES) (pH 7.9), $10 \mathrm{mM} \mathrm{KCl,} \mathrm{0.1} \mathrm{mM} \mathrm{EDTA,} 0.1 \mathrm{mM}$ EGTA, $1 \mathrm{mM}$ DTT and $0.5 \mathrm{mM}$ PMSF. After $15 \mathrm{~min}$ on ice, the cells were vortexed in the presence of $0.5 \%$ Nonidet NP-40. The nuclear pellet was then collected by centrifugation and extracted in a buffer containing $20 \mathrm{mM}$ HEPES ( $\mathrm{pH} 7.9$ ), $0.4 \mathrm{M} \mathrm{NaCl}, 1 \mathrm{mM}$ EDTA, $1 \mathrm{mM}$ EGTA, $1 \mathrm{mM}$ DTT and $1 \mathrm{mM}$ PMSF for $15 \mathrm{~min}$ at $4^{\circ} \mathrm{C}$.

The nuclear extract $(10-20 \mu \mathrm{g})$ was preincubated at $4^{\circ} \mathrm{C}$ for 30 min with a 100 -fold excess of an unlabeled oligonucleotide spanning the -79 MMP-9 cis element of interest. The sequences were as follows: AP-1, CTGACCCCTGAGTCAGCACTT; NF- $\kappa$, CAGTGGAATTCCCCAGCC; Sp-1, GCCCATTC CTTCCGCCCCCAGATGAAGCAG. The reaction mixture was then incubated at $4^{\circ} \mathrm{C}$ for $20 \mathrm{~min}$ in a buffer $(25 \mathrm{mM}$ HEPES buffer $\mathrm{pH} 7.9,0.5 \mathrm{mM}$ EDTA, $0.5 \mathrm{mM}$ DTT, $0.05 \mathrm{M}$ $\mathrm{NaCl}$, and $2.5 \%$ glycerol) with $2 \mu \mathrm{g}$ of poly $\mathrm{dI} / \mathrm{dC}$ and $5 \mathrm{fmol}$ $\left(2 \times 10^{4} \mathrm{cpm}\right)$ of a Klenow end-labeled $\left({ }^{32} \mathrm{P}\right.$-ATP) 30 -mer 

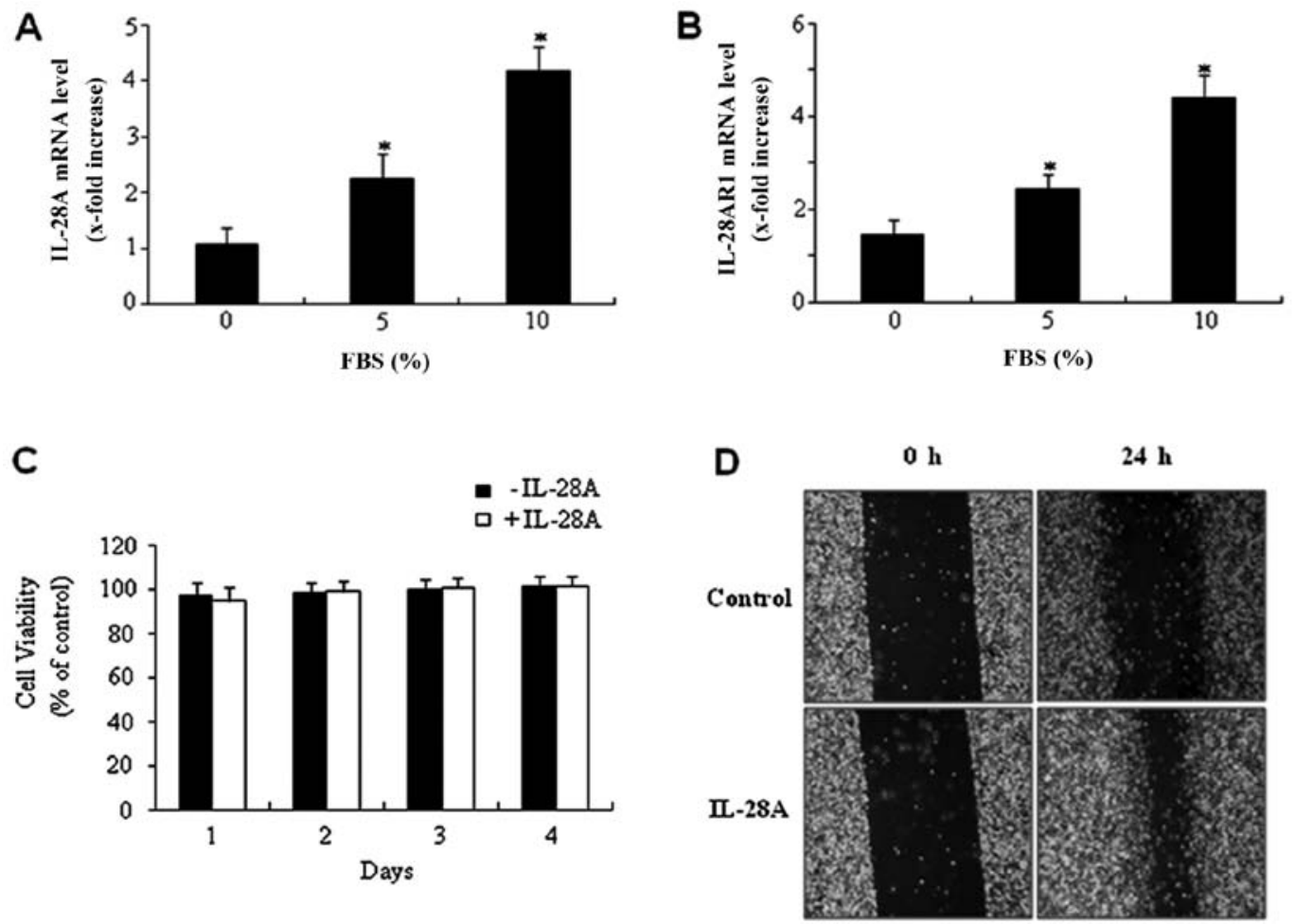

Figure 1. IL-28A treatment of UMUC-3 cells induces wound-healing migration. Expression of (A) IL-28A and (B) its receptor IL-28AR1 in UMUC-3 cells. Cells were grown to 70\% confluence in DMEM supplemented with 10\% FBS. After serum starvation for $24 \mathrm{~h}$, the cells were treated with or without the indicated concentrations of $10 \%$ FBS for $24 \mathrm{~h}$. Expression of mRNA levels of IL-28A and IL-28AR1 was analyzed by real-time PCR. "P<0.01 compared with no treatment. (C) IL-28A had no effect on cell proliferation as determined by the MTT assay. After serum starvation for $24 \mathrm{~h}$, cells were cultured for 1 to 4 days in serum-free medium supplemented with IL-28A $(100 \mathrm{ng} / \mathrm{ml})$. MTT assay was performed. (D) After serum starvation for $24 \mathrm{~h}$, cells were cultured with IL-28A (100 ng/ml) for $24 \mathrm{~h}$. Wound-healing assay revealed an evident increase in the wound closure rates of UMUC-3 cells after IL-28A (100 ng/ml) stimulation for $24 \mathrm{~h}$.

oligonucleotide, which spanned the DNA binding site in the MMP-9 promoter. The reaction mixture was electrophoresed at $4^{\circ} \mathrm{C}$ in a $6 \%$ polyacrylamide gel using a TBE $(89 \mathrm{mM}$ Tris, $89 \mathrm{mM}$ boric acid and $1 \mathrm{mM}$ EDTA) running buffer. The gel was rinsed with water, dried, and exposed to X-ray film overnight (15).

Statistical analysis. Where it was appropriate, data were expressed as the mean \pm SE. Data were analyzed by factorial ANOVA and Fisher's least significant difference test where appropriate. Statistical significance was set at $\mathrm{P}<0.05$.

\section{Results}

Expression of IL-28A and IL-28ARI in UMUC-3 bladder cancer cells. To determine the expression levels of IL-28A and its receptor IL-28AR1, real-time PCR assay was performed on bladder cancer UMUC-3 cells. Expression of IL-28A mRNA was detected in UMUC-3 cells (Fig. 1A). In addition, IL-28AR1 mRNA was also found to be expressed in UMUC-3 cells (Fig. 1B). Exposure of UMUC-3 cells to $10 \%$ FBS for $24 \mathrm{~h}$ markedly increased the IL-28A mRNA expression compared with the non-treated cells (Fig. 1A). A similar result was observed in the expression level of IL-28AR1 mRNA (Fig. 1B). These results indicated that both IL-28A and IL-28AR1 mRNA were expressed in the UMUC-3 bladder cancer cells.
IL-28A induces migration of UMUC-3 bladder cancer cells. MTT assay was used to assess the proliferative ability of IL-28A. IL-28A treatment did not affect the levels of cell proliferation (Fig. 1C). Subsequently, a wound-healing migration assay was carried out to determine whether IL-28A induces cell migration. Monolayer UMUC-3 cells were scratched, and the wounds were allowed to heal for $24 \mathrm{~h}$. Cell migration ability at the wound front was analyzed and compared to the initial wound state. UMUC-3 cells treated with IL-28A for $24 \mathrm{~h}$ showed a significant increase in cell migration (Fig. 1D). These results suggest that IL-28A induces migration of UMUC-3 cells in vitro.

IL-28A stimulates MMP-9 expression via binding activation of $N F-\kappa B$ and $A P-1$. Since cell migration is associated with matrix degradation by MMPs (7-9), we next examined the expression of MMPs in the UMUC-3 cell culture supernatants using zymographic analysis (Fig. 2A). To determine the effect of IL-28A on the expression of MMPs, cells were treated with IL-28A at the indicated concentrations for the indicated times. Treatment with IL-28A induced MMP-9 expression in UMUC-3 cells in a concentration- and a time-dependent manner (Fig. 2A). These results were confirmed by immunoblot analysis using an antibody that detects a band of MMP-9 (Fig. 2A). In addition, MMP-2 levels were also elevated in UMUC-3 cells following IL-28A treatment (Fig. 2A). Previous studies showed that enhanced expression of MMP-9 is directly correlated with 
A
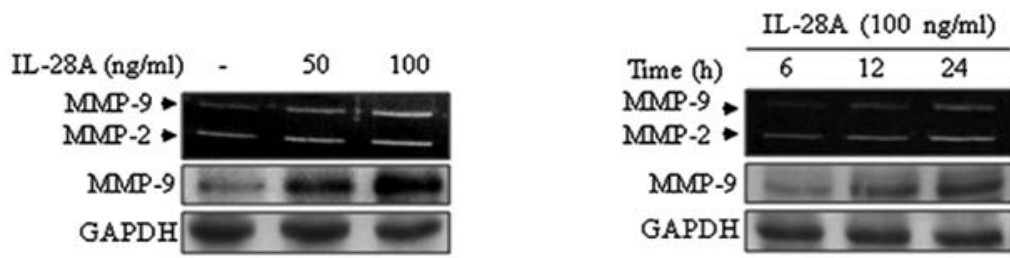

B
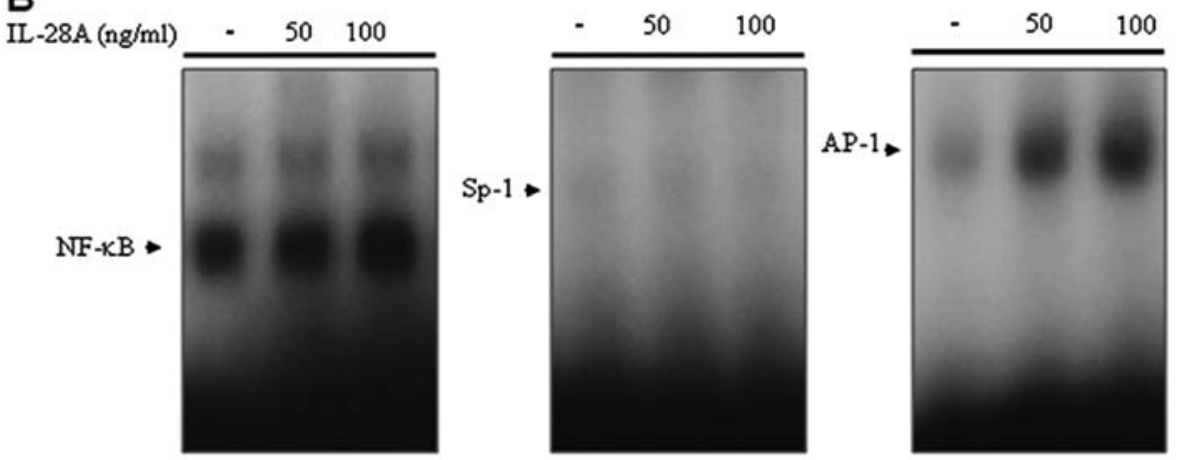

Figure 2. IL-28A induces MMP-9 expression via binding activities of NF- $\mathrm{kB}$ and AP-1 motifs in UMUC-3 cells. (A) After serum starvation for $24 \mathrm{~h}$, the cells were treated with the indicated concentrations of IL-28A and then further incubated for $24 \mathrm{~h}$. The proteolytic activity of MMP-9 in the supernatants was determined using gelatin zymography. The protein levels of MMP-9 from the lysates were analyzed by immunoblotting. (B) After serum starvation for $24 \mathrm{~h}$, cells were treated with the indicated concentrations of IL-28A for $24 \mathrm{~h}$. EMSA was performed using individual ${ }^{32} \mathrm{P}-$ labeled probes containing binding motifs of various putative transcription factors NF-kB, AP-1 and Sp-1 on the 5' flanking region of MMP-9.
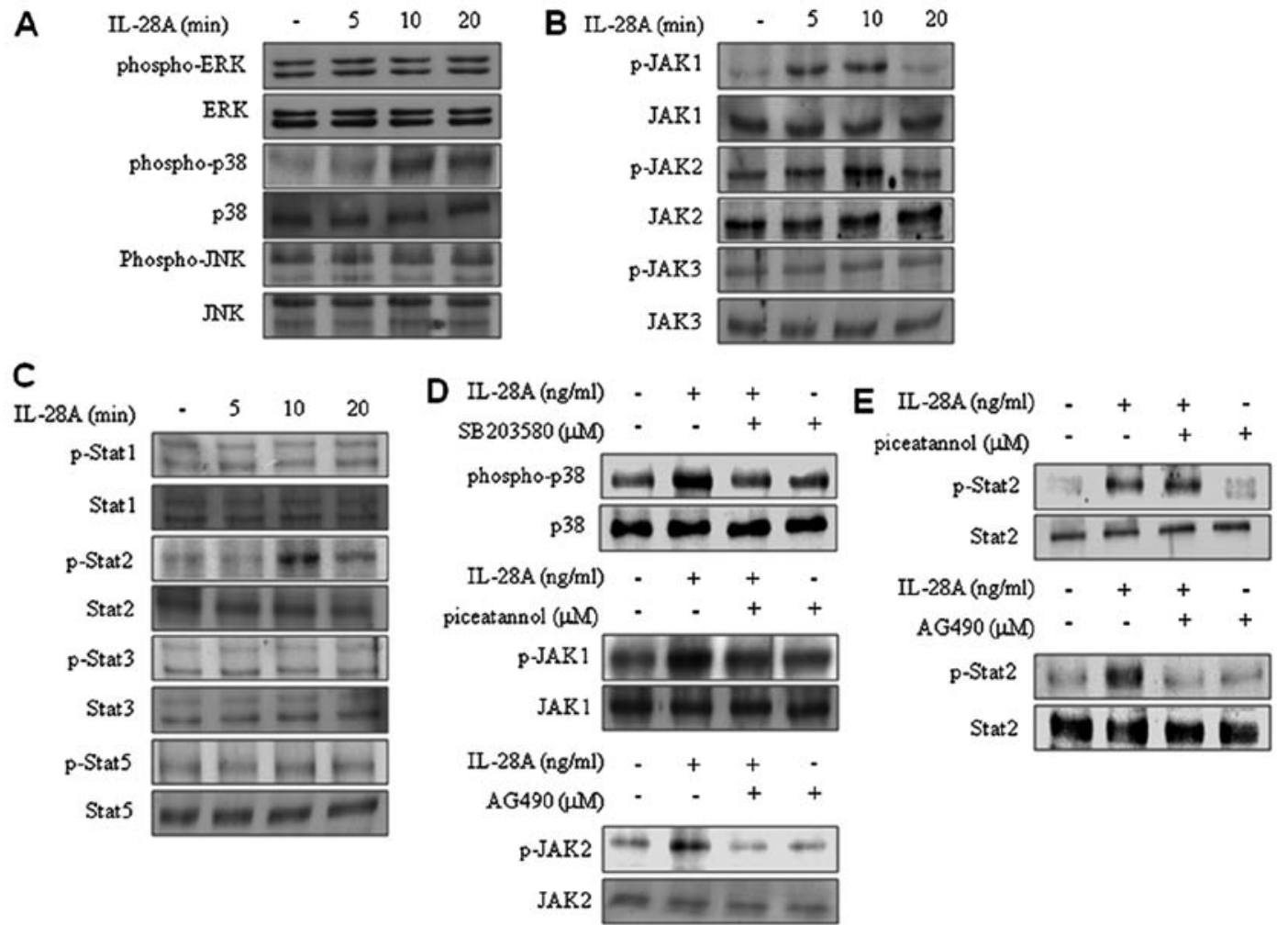

Figure 3. IL-28A induces activation of p38 MAPK and Jak2-Stat2 signaling. (A-C) After serum starvation for $24 \mathrm{~h}$, cells were incubated with IL-28A $(100 \mathrm{ng} / \mathrm{ml})$ for the indicated time intervals, and activation of ERK1/2, p38 MAPK, JNK, Jak1, Jak2, Jak3, Stat1, Stat2, Stat3 and Stat5 was determined by immunoblot analysis. (D and E) After serum starvation for $24 \mathrm{~h}$, the cells were pretreated with p38 MAPK inhibitor SB203580, JAK1 inhibitor piceatannol, and Jak2 inhibitor AG490 for 40 min prior to treatment with IL-28A (10 ng/ml), and were then further incubated for 10 min. Activation of p38 MAPK, Jak1, Jak2 and Stat 2 was analyzed by immunoblotting. The results represent three independent experiments.

the progression of bladder cancer characterized by increased tumor grade, invasion and metastasis $(10,11)$. Therefore, we focused on MMP-9 regulation induced by IL-28A in UMUC-3 cells. To do this, we investigated MMP-9 regulation at the transcriptional level using transcription factors NF- $\mathrm{BB}, \mathrm{AP}-1$ and Sp-1, all of which were located in the $5^{\prime}$ flanking region of 

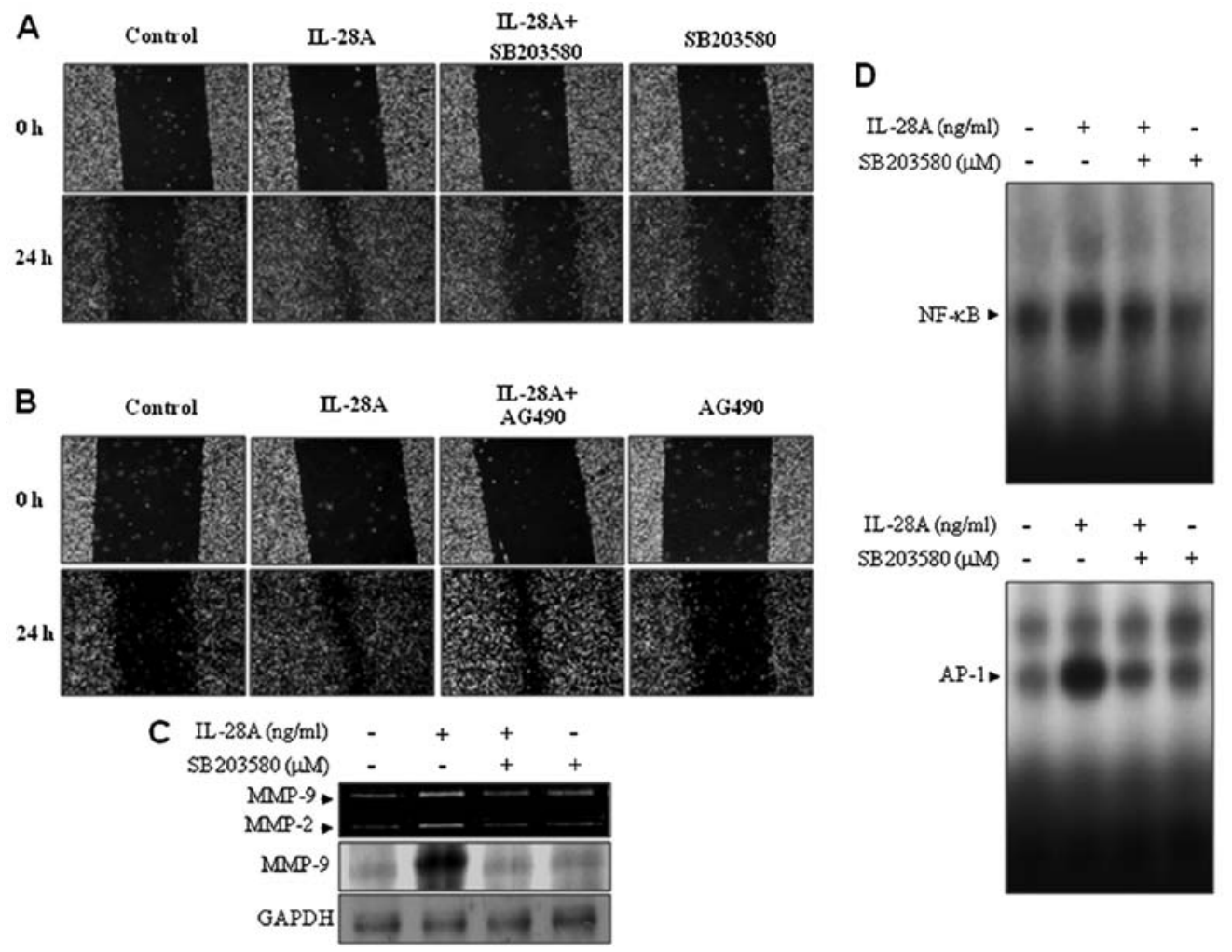

Figure 4. Effects of SB203580 on wound-healing migration, MMP-9 expression, and binding activities of NF-kB and AP-1 in IL-28A-treated UMUC-3 cells. After serum starvation for $24 \mathrm{~h}$, cells were pre-incubated with (A) SB203580 (10 $\mu \mathrm{M})$ or (B) AG490 (10 $\mu \mathrm{M})$ for 40 min and then stimulated by IL-28A (100 ng/ $\mathrm{ml}$ ) for $24 \mathrm{~h}$, and wound-healing migration assay was performed as described in Materials and methods. (C) The cells were treated with IL-28A (100 ng/ml) in the presence or absence of SB203580 $(10 \mu \mathrm{M})$. MMP-9 expression was analyzed in the culture medium using zymographic assay. Immunoblot assay was used to determine the protein levels of MMP-9 in the cell lysates. (D) EMSA analyses were performed on nuclear extracts for activated NF- $\mathrm{kB}$ and AP-1 using radiolabeled oligonucleotide probes.

the MMP-9 gene (12-15). Our results from EMSA showed that IL-28A treatment enhanced binding activation of NF- $\mathrm{KB}$ and AP-1 (Fig. 2B). However, no specific binding activation of Sp-1 was detected in the IL-28A-induced UMUC-3 cells (Fig. 2B). These results demonstrate that IL-28A induced MMP-9 expression through binding motifs of the transcription factors NF- $\kappa \mathrm{B}$ and AP-1.

IL-28A induces the activation of $p 38$ MAPK and Jak2-Stat2 signaling. To determine the signaling pathway in IL-28Atreated UMUC-3 cells, we examined the MAPK signaling pathway. Treatment with IL-28A significantly induced activation of p38 MAPK in UMUC-3 cells (Fig. 3A). Pretreatment with SB203580 inhibited IL-28A-stimulated activation of p38 MAPK (Fig. 3D). In contrast, activation of both ERK1/2 and JNK was not affected in IL-28A-treated cells (Fig. 3A). We also observed the effects of IL-28A on the activation of Jak-Stat signaling. IL-28A treatment increased activation of Jak1, Jak2, and Stat2. However, activation of Jak3, Stat1, Stat3 and Stat5 was not affected by IL-28A treatment (Fig. 3B and C). To confirm the activation of Jak1 and Jak2 signaling, we used Jak1 inhibitor (piceatannol) and Jak2 inhibitor (AG490). The cells were pretreated with piceatannol or AG490, respectively, and then further incubated with IL-28A treatment for $10 \mathrm{~min}$. IL-28A-induced activation of Jak1 was blocked by piceatannol (Fig. 3D). AG490 also abolished activation of Jak2 in IL-28Atreated UMUC-3 cells (Fig. 3D). Next, we further investigated whether Jak1 or Jak2 was involved in IL-28A-induced Stat2 activation. Inhibition of Jak2 by AG490 suppressed the Stat2 activation that was induced by IL-28A (Fig. 3E). However, piceatannol treatment had no apparent effect on the IL-28Astimulated activation of Stat2 (Fig. 3E). These results suggest that p38 MAPK and Jak2-Stat2 signaling are involved in IL-28A-treated UMUC-3 cells.

Involvement of SB203580, a p38 MAPK inhibitor, in woundhealing migration, MMP-9 expression, and binding activities of NF- $\kappa B$ and AP-1 in IL-28A-treated UMUC-3 cells. We next investigated which signaling pathway, p38 MAPK and/ or Jak2, mediates the enhanced migration of IL-28A-treated UMUC-3 cells. Pretreatment of cells with SB203580 inhibited the IL-28A-induced migration of UMUC-3 cells (Fig. 4A). However, the addition of AG490 had no effect on the cell migration induced by IL-28A (Fig. 4B). These results indicate that $\mathrm{p} 38$ MAPK signaling may contribute to the induction of UMUC-3 cell migration. The results obtained above prompted us to examine whether the p38 MAPK signaling pathway is involved in IL-28A-mediated MMP-9 regulation. UMUC cells were treated with SB203580 in the presence or absence of IL-28A. The results from zymographic and immunoblot analyses showed that pretreatment with SB203580 significantly attenuated IL-28A-induced MMP-9 expression (Fig. 4C). To analyze the role of $\mathrm{p} 38$ MAPK in the transcriptional mechanism of MMP-9 in IL-28A-treated cells, we performed EMSA on the NF- $\kappa$ B and AP-1 binding sites from the MMP-9 promoter using nuclear extracts. SB203580 treatment 
A

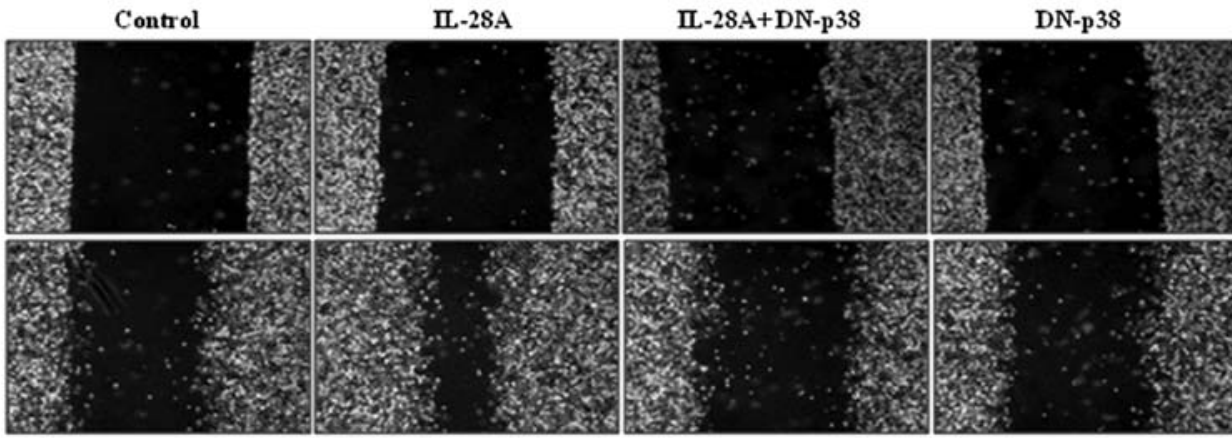

B
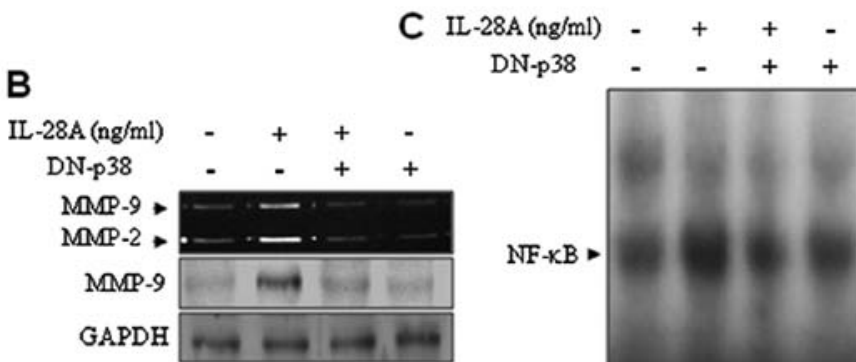

$\mathrm{DN}-\mathrm{p} 38 \quad-\quad+\quad+$
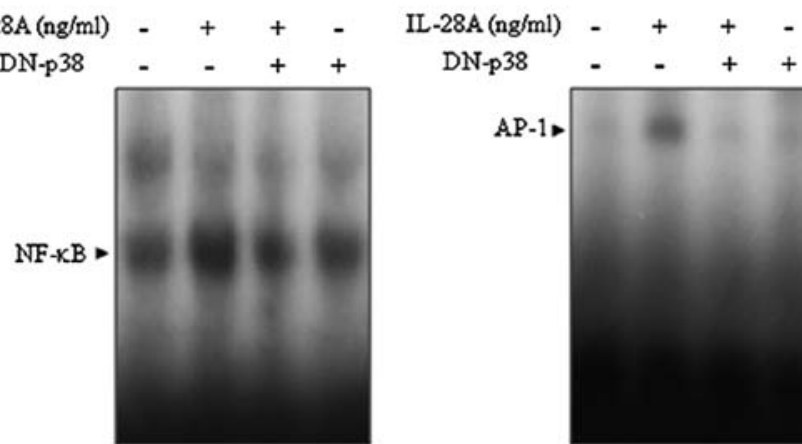

DN-p38

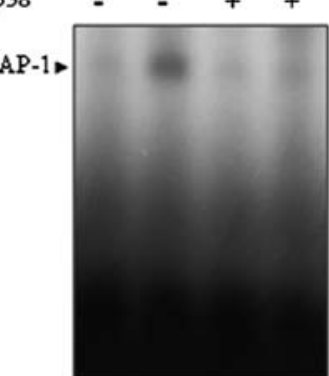

Figure 5. Effects of DN-p38 on wound-healing migration, MMP-9 expression, and binding activities of NF- $\kappa$ B and AP-1 motifs in the IL-28A-stimulated UMUC-3 cells. (A) Cells were transfected with DN-p38 for $24 \mathrm{~h}$ and then stimulated by IL-28A (100 ng/ml). The wound-healing migration was measured after $24 \mathrm{~h}$ as described in Materials and methods. (B) Cells were transfected with DN-p38 for $24 \mathrm{~h}$, followed by stimulation with IL-28A. After $24 \mathrm{~h}$, the expression of MMP-9 was determined in the culture medium using zymographic analysis, and the MMP-9 protein levels in the cell lysates were determined using an immunoblot assay. (C) Nuclear extracts from the cells were analyzed by EMSA to determine the binding activities of NF- $\kappa$ B and AP-1 motifs using radiolabeled oligonucleotide probes.

inhibited the induction of binding activities of $\mathrm{NF}-\kappa \mathrm{B}$ and AP-1 (Fig. 4D). These results demonstrate that p38 MAPK signaling is involved in cell migration and MMP-9 expression via the binding activities of NF- $\kappa \mathrm{B}$ and AP-1 in IL-28A-treated UMUC-3 cells

Effect of a dominant-negative p38 MAPK mutant gene on wound-healing migration, MMP-9 expression, and binding activities of $N F-\kappa B$ and AP-1 in UMUC-3 cells. To confirm the direct roles of p38 MAPK in IL-28A-mediated cellular responses, DN-p38, a dominant-negative p38 MAPK mutant gene, was tested. To this end, UMUC-3 cells were transfected with DN-p38 plasmid and then treated with IL-28A. DN-p38 transfectant inhibited the wound-healing migration of UMUC-3 cells induced by IL-28A (Fig. 5A). In addition, IL-28A-induced MMP-9 expression was suppressed in the DN-p38-transfected cells (Fig. 5B). Finally, overexpression of DN-p38 almost abolished increased NF- $\mathrm{KB}$ and AP-1 DNA binding activities in the presence of IL-28A (Fig. 5C). Together, these results indicate that p38 MAPK signaling is required for cell migration via MMP-9 expression by inducing activation of NF- $\mathrm{NB}$ and AP-1 binding in IL-28A-treated UMUC-3 cells.

\section{Discussion}

There is much evidence linking tumor progression and development to inflammatory cytokines that are produced by tumor cells $(23,24)$. The main inflammatory cytokines include IL-1 $\beta$, IL-6, IL-23 and TNF- $\alpha$, which are also associ- ated with the tumorigenic and metastatic potential of several cancer cells $(23,24)$. Previous studies have demonstrated that pro-inflammatory cytokines such as TNF- $\alpha$, IL-6 and IL-8 are putative mediators of metastasis in bladder cancer cells (2,25-28). However, there has been no report of the functional role of IL-28A in tumor migration. In the present study, we report the role of IL-28A in the migration of UMUC-3 bladder cancer cells.

IL-28A is a human IFN- $\lambda 2$ protein with pro-inflammatory and antiviral activity $(17,18)$. Despite all of these functions, the migratory mechanisms of IL-28A produced by tumor cells are unclear. In this study, we found that UMUC-3 bladder cancer cells produced IL-28A and its receptor IL-28AR1 as determined by real-time PCR. We next examined whether IL-28A is involved in the migration of bladder cancer cells. The exogenous IL-28A enhanced in vitro migration of UMUC-3 cells without altering cell proliferation, suggesting that IL-28A induces bladder cancer cell migration. These results contradict other reports which have suggested that IL-28A exhibits an antitumor effect against B16 melanoma and murine fibrosarcoma $(21,22)$. Further study is required to verify the in vivo efficacy of the IL-28A gene in bladder tumor migration.

MMPs degrade various components of the extracellular matrix (ECM) and basement membrane proteins that are considered to be essential in tumor migration (7-9). Gelatinases (MMP-2 and -9) have been connected to the metastatic potential of malignant tumors (7-9). The expression of MMP-9 is directly correlated with bladder tumor progression characterized by increased tumor grade, invasion and metastasis $(10,11)$. Our results showed that exposure to IL-28A induced MMP-9 
expression in UMUC-3 cells. The MMP-9 promoter contains a proximal AP-1 element, an upstream Sp-1, and a NF- $\mathrm{B}$ binding motif in tumor cell lines (12-15). Previous reseach has demonstrated that the transcription factors NF- $\mathrm{B}, \mathrm{AP}-1$ and Sp-1 are associated with the regulation of TNF- $\alpha$-stimulated MMP-9 in bladder cancer 5637 and HT1376 cells $(27,28)$. However, the identification of cis elements in the induction of MMP-9 in response to IL-28A in bladder cancer is unclear. The results from EMSA indicated that NF- $\kappa$ B and AP-1 binding sites within the MMP-9 promoter region are crucial for IL-28A-stimulated MMP-9 expression. These data suggest that IL-28A may promote cell migration through the induction of MMP-9 by activating NF- $\kappa \mathrm{B}$ and AP-1 binding to the promoter.

Inflammatory cytokine signals of IL-28A have been shown to induce activation of Stat1, Stat 2 and Stat 3 in HT-29 cells expressing IL-28AR1 (17). In addition, IL-28A treatment was found to decrease cell proliferation in colon cancer cell lines, which was followed by activated signaling of ERK1/2, JNK, AKT and Stat-1 (19). We examined the signaling pathway induced by IL-28A in UMUC-3 bladder cancer cells. The addition of IL-28A induced activation of p38 MAPK and Jak2Stat 2 signaling in UMUC-3 cells. Several signaling pathways, ncluding p38 MAPK and Jak-Stat signaling, are known to be involved in cell migration and MMP-9 regulation in various cell types $(12,16)$. Our results showed that pretreatment with the p38 MAPK inhibitor, SB203580, significantly blocked IL-28A-induced migration and MMP-9 expression. However, unexpectedly, inhibition of cell migration by Jak2 inhibitor AG490 was not observed in IL-28A-treated UMUC-3 cells. We also found that the involvement of p38 MAPK in IL-28Ainduced migration and MMP-9 expression was revealed by transfection with DN-p38. Finally, we identified the transcription factors that are involved in the p38 MAPK-mediated control of MMP-9 expression in UMUC-3 cells in the presence of IL-28A using SB203580 and the DN-p38 plasmid. The present study revealed that the ability of SB203580 and DN-p38 to reduce MMP-9 expression in IL-28A-treated UMUC-3 cells was accomplished by suppressing NF- $\mathrm{B}$ and AP-1 binding. Collectively, our results demonstrated that p38 MAPK signaling by IL-28A participated in MMP-9 expression by activating NF- $\mathrm{B}$ and AP-1 binding resulting in the degradation of ECM and leading to the migration of bladder cancer cells.

Cytokines have the potential to stimulate the proliferation and migration of malignant cells. However, the relationship between cytokines and tumor progression has been controversial $(23,24)$. In recent years, many researchers have studied molecular mechanisms by which cytokines may act to promote tumor development. In the present study, we demonstrated that IL-28A induces the migration of bladder cancer cells via the degradation of ECM components by proteolytic enzymes such as type IV collagenase. Previous studies have suggested an opposing role for IL-28A, i.e., that it exhibits an antitumor effect in tumor systems in vitro and in vivo $(21,22)$. Future study is required to clarify the biological action of IL-28A on different tumor cells.

In conclusion, we introduced a novel mechanism by which p38 MAPK is responsible for IL-28A-induced migration of UMUC-3 bladder cancer cells, which is associated with
MMP-9 expression via the binding activities of NF- $\kappa \mathrm{B}$ and AP-1. Therefore, IL-28A is a potential therapeutic target for suppressing the metastasis of bladder tumor cells.

\section{Acknowledgements}

This research was supported by the Basic Science Research Program through the National Research Foundation of Korea (NRF), funded by the Ministry of Education, Science and Technology (2012-0000482). This research was also supported by a grant from the Academic Research Program of Korea National University of Transportation in 2012.

\section{References}

1. Jemal A, Siegel R, Ward E, Murray E, Xu T and Thun MJ: Cancer statistics. CA Cancer J Clin 57: 43-66, 2007.

2. Black PC and Dinney CP: Bladder cancer angiogenesis and metastasis - translation from murine model to clinical trial. Cancer Metastasis Rev 26: 623-634, 2007.

3. Metts MC, Metts JC, Milito SJ and Thomas CR Jr: Bladder cancer: a review of diagnosis and management. J Natl Med Assoc 92: 285-294, 2000.

4. Crawford JM: The origins of bladder cancer. Lab Invest 88: 686-693, 2008

5. Heney NM, Proppe K, Prout GR, Griffin PP and Shipley WU: Invasive bladder cancer: tumor figuration, lymphatic invasion and survival. J Urol $130: 895-897,1983$.

6. Mitra AP, Bartsch CC and Cote RJ: Strategies for molecular expression profiling in bladder cancer. Cancer Metastasis Rev 28: 317-326, 2009.

7. Stetler-Stevenson WG, Aznavoorian S and Liotta LA: Tumor cell interactions with the extracellular matrix during invasion and metastasis. Annu Rev Cell Biol 9: 541-573, 1993.

8. Matrisian LM: Metalloproteinases and their inhibitors in matrix remodeling. Trends Genet 6: 121-125, 1990

9. Westermarck J and Kahari VM: Regulation of matrix metalloproteinase expression in tumor invasion. FASEB J 13: 781-792, 1999.

10. Davies B, Waxman J, Wasan H, Abel P, Williams G, Krausz T, Neal D, Thomas D, Hanby A and Balkwill F: Levels of matrix metalloproteases in bladder cancer correlate with tumor grade and invasion. Cancer Res 53: 5365-5369, 1993.

11. Bianco FJ, Gervasi DC Jr, Tiguert R, Grignon DJ, Pontes JE, Crissman JD, Fridman R and Wood DP Jr: Matrix metalloproteinase-9 expression in bladder washes from bladder cancer patients predicts pathological stage and grade. Clin Cancer Res 4: 3011-3016, 1998

12. Mook OR, Frederiks WM and Van Noorden CJ: The role of gelatinases in colorectal cancer progression and metastasis. Biochim Biophys Acta 1705: 69-89, 2004.

13. Bond M, Rosalind P, Fabunmi P, Baker AH and Newby AC: Synergistic upregulation of metalloproteinase- 9 by growth factors and inflammatory cytokines: an absolute requirement for transcription factor NF-kappa B. FEBS Lett 435: 29-34, 1998.

14. Sato $\mathrm{H}$ and Seiki M: Regulatory mechanism of $92 \mathrm{kDa}$ type IV collagenase gene expression which is associated with invasiveness of tumor cells. Oncogene 8: 395-405, 1993.

15. Moon SK, Cha BY and Kim CH: ERK1/2 mediates TNF-alphainduced matrix metalloproteinase- 9 expression in human vascular smooth muscle cells via the regulation of NF-kappaB and AP-1: Involvement of the ras dependent pathway. J Cell Physiol 198: 417-427, 2004

16. Kim S, Choi JH, Lim HI, Lee SK, Kim WW, Cho S, Kim JS, Kim JH, Choe JH, Nam SJ, et al: EGF-induced MMP-9 expression is mediated by the JAK3/ERK pathway, but not by the JAK3/STAT-3 pathway in a SKBR3 breast cancer cell line. Cell Signal 21: 892-898, 2009.

17. Kotenko SV, Gallagher G, Baurin VV, Lewis-Antes A, Shen M, Shah NK, Langer JA, Sheikh F, Dickensheets H and Donnelly RP: IFN- $\lambda$ s mediate antiviral protection through a distinct class II cytokine receptor complex. Nat Immunol 4: 69-77, 2003.

18. Sheppard P, Kindsvogel W, Xu W, Henderson K, Schlutsmeyer S, Whitmore TE, Kuestner R, Garrigues U, Birks C, Roraback J, et al: IL-28, IL-29 and their class II cytokine receptor IL-28R. Nat Immunol 4: 63-68, 2003. 
19. Brand S, Beigel F, Olszak T, Zitzmann K, Eichhorst ST, Otte JM, Diebold J, Diepolder H, Adler B, Auernhammer CJ, et al: IL-28A and IL-29 mediate antiproliferative and antiviral signals in intestinal epithelial cells and murine CMV infection increases colonic IL-28A expression. Am J Physiol Gastrointest Liver Physiol 289: G960-G968, 2005.

20. Yang L, Luo Y, Wei J and He S: Integrative genomic analyses on IL28RA, the common receptor of interferon- $\lambda 1,-\lambda 2$ and $-\lambda 3$. Int J Mol Med 25: 807-812, 2010.

21. Lasfar A, Lewis-Antes A, Smirnov SV, Anantha S, Abushahba W, Tian B, Reuhl K, Dickensheets H, Sheikh F, Donnelly RP, et al: Characterization of the mouse IFN- $\lambda$ ligand-receptor system: IFN- $\lambda$ s exhibit antitumor activity against B16 melanoma. Cancer Res 66: 4468-4477, 2006.

22. Numasaki M, Tagawa M, Iwata F, Suzuki T, Nakamura A, Okada M, Iwakura Y,Aiba S and Yamaya M: IL-28 elicits antitumor responses against murine fibrosarcoma. J Immunol 178: 5086-5098, 2007.

23. Coussens LM and Werb Z: Inflammation and cancer. Nature 420: $860-867,2002$
24. Mantovani A, Allavena P, Sica A and Balkwill F: Cancer-related inflammation. Nature 454: 436-444, 2008.

25. Okamoto $\mathrm{M}$, Hattori $\mathrm{K}$ and Oyasu R: Interleukin-6 functions as an autocrine growth factor in human bladder carcinoma cell lines in vitro. Int J Cancer 72: 149-154, 1997.

26. Inoue K, Slaton JW, Kim SJ, Perrotte P, Eve BY, Bar-Eli M, Radinsky R and Dinney CP: Interleukin 8 expression regulates tumorigenicity and metastasis in human bladder cancer. Cancer Res 60: 2290-2299, 2000.

27. Lee SJ, Park SS, Cho YH, Park K, Kim EJ, Jung KH, Kim SK, Kim WJ and Moon SK: Activation of matrix metalloproteinase-9 by TNF- $\alpha$ in human urinary bladder cancer HT1376 cells: The role of MAP kinase signaling pathways. Oncol Rep 19: 1007-1013, 2008.

28. Lee SJ, Park SS, Lee US, Kim WJ and Moon SK: Signaling pathway for TNF-alpha-induced MMP-9 expression: mediation through p38 MAP kinase, and inhibition by anti-cancer molecule magnolol in human urinary bladder cancer 5637 cells. Int Immunopharmacol 8: 1821-1826, 2008. 